\title{
Convection Rolls and Individual Particles Movements in Horizontally Vibrated Granular Particles System
}

\author{
A. Rehman ${ }^{a}$, Ping Wu ${ }^{a, *}$, Li Li ${ }^{a}$, Shiping Zhang ${ }^{a}$ AND Li WANG ${ }^{b}$ \\ ${ }^{a}$ School of Mathematics and Physics, University of Science and Technology, Beijing, 10083, China \\ ${ }^{b}$ School of Energy and Environmental Engineering, University of Science and Technology, Beijing, 10083, China
}

(Received July 15, 2016)

\begin{abstract}
Convection in horizontally vibrated granular systems is significant for scientists and engineers for their importance in the field of mining, geo-physics, and pharmaceutical etc. This research work studied three types of convection rolls, "Homogeneous convection roll", "lower-right diagonal convection roll" and "upper-right diagonal convection roll" which occurred in a square container filled with binary granular particles mixture of sized $d=(4.0 \pm 0.2) \mathrm{mm}$ and $d=(8.0 \pm 0.2) \mathrm{mm}$. Container was vibrated horizontally with low frequencies $f$ and low dimensionless acceleration $\Gamma$. Helical movement was observed along the walls perpendicular to direction of motion while straight-line movement along the walls horizontal to direction of motion. Helical and straight-line movements of particles along the walls are the part of convection rolls. A heap appeared due to vibration, which has dominant effect on the convection rolls. Heap position is function of frequency $f$ and dimensionless accelerations $\Gamma$.
\end{abstract}

DOI: 10.12693/APhysPolA.130.1336

PACS/topics: 45.70.-n, 47.57.Gc, 47.52.+j, 47.57.Qk, 83.80.Fg

\section{Introduction}

Study of granular materials is significant and important because they have apparent solid-like properties but behave like a fluid in certain conditions. Behaviors of granular material are excessively difficult to understand because Newtonian mechanics and fluid dynamics cannot employ directly. The Brazilian nut effect (BNE) is one such system that has been studied much experimentally $[1-5]$ and theoretically $[6-9]$. Similar behaviors detected on horizontal swirling motion in circular plates [10] large bead will migrate either to the center or to the border of the plate. In this horizontal version of the BNE system another interesting phenomenon was reported [11], in which the spin angular velocity of the cluster of beads decreases with increasing packing density. Even more amazingly, at a certain critical packing density, the angular velocity becomes negative, i.e., the cluster and the plate rotate in opposite directions.

Convection in deep horizontally shaken systems reveals a complex structure [12-15] and it is continuous issue of exciting research [16-18]. Convection behavior gives rise to a "reverse-muesli" effect, where large particles placed at the top of the pill will move downward until it reaches the upwelling of the lower convection rolls. For small heaps the large particle will actually reach the ground, and surface wave convection may cause a large particle to return to the top of the pile [19]. Spontaneous formation of a static heap and convection flow depends on $\Gamma$ and phase difference $\Phi[20]$. In rectangular container submitted linear horizontal vibration there may be observed two convection rolls; the grains rise at the cell center and dive along the vertical walls [21]. Various types

\footnotetext{
* corresponding author; e-mail: pingwu@sas.ustb.edu.cn
}

of convection cells, convection velocity measurement and mechanisms responsible for different type of motions were discussed [22]. Horizontally oscillated granular materials showed four rolls convection patterns, two small convective rolls and two lower internal rolls produced by surface swilling. The mechanism was different from vertically vibrated systems and convection patterns can drive size segregation [23]. Roughness of the boundaries and the container sizes were the influencing factors that affected the shape and number of convection rolls [24]. New mechanisms might be expected which are fundamentally different from those responsible for the convective behavior in vertically vibrated systems [12]. Simulations also discuss convection patterns in horizontally shaken containers. Molecular dynamics simulations of horizontally vibrated granular materials also showed that normal damping coefficient affects the convection patterns [25]. Two cylindrical counter-rotating rolls were observed at low dimensionless acceleration [26]. Convection rolls were combined with segregation [27]. Convective motion of walldriven granular systems was investigated [28]. Height variations, strength of the convection movement and energy dissipation related to each other [29]. Hysteresis of the transition explained through energy of collective movement of particles relative to the container [30]. Various states, phase transitions of processes of convection and segregation were analyzed by specifying the earlier unnoticed dynamical phenomena [31]. Mechanics behind the homogeneous and non-homogeneous convection in granular systems is important for scientists and engineers for industrial processes. This paper discusses three different types of convection rolls experimentally and individual particles movement at low frequency and low dimensionless acceleration in horizontally vibrated granular particle system. 


\section{Experimental setup}

This experimental work was performed in a square container of $25 \mathrm{~cm}$ by sides and $18 \mathrm{~cm}$ by height. The container was made of transparent material in order to make it visible with the aid of high-speed camera. The base and side walls of container were smooth. The experiment was carried out on smooth binary granular materials of $d=(4.0 \pm 0.2) \mathrm{mm}$ and $d=(8.0 \pm 0.2) \mathrm{mm}$ sized active aluminum oxide particles with density of $\rho=1.6 \mathrm{~g} / \mathrm{cm}^{3}$. The container was filled with $1000 \mathrm{~g}$ of $d=(4.0 \pm 0.2) \mathrm{mm}$ sized particles and $1000 \mathrm{~g}$ of $d=(8.0 \pm 0.2) \mathrm{mm}$ sized particles. The filling height of particles was $h=30.0 \mathrm{~mm}$. Horizontal sinusoidal motion $x(t)=A \sin (2 \pi f t)$ of the container was provided by a vibration testing systems, the electromagnetic shaker and a function generator. Three frequencies $5,10,15 \mathrm{~Hz}$ were used in experiment and acceleration $a=A(2 \pi f)^{2}$ changed from 1 to $15 \mathrm{~m} / \mathrm{s}^{2}$ for each frequency. The magnitude of the acceleration $a$ was measured by a UNIT (UT311) pocketable accelerometer mounted directly on the vibrator. The convection patterns were observed through transparent walls by using a strobe light synchronized to the driving frequency. Canon Motion Carder video system was used which is able to record images 500 frames/s. This system allowed us to store large numbers of frames taken with very short exposure times at the same phase of consecutive cycles and from such data, the convection patterns were observed, and the convection flow were computed.

\section{Results}

The system vibrated horizontally with 5,10 , and $15 \mathrm{~Hz}$ frequencies and dimensionless acceleration $\Gamma$ varied from 0.1 to 1.4 for each frequency. Dimensionless acceleration is $\Gamma=A \omega^{2} / g$, where $A$ is amplitude of vibration, $\omega$ is angular velocity and $g$ is gravitational acceleration. Before the critical value of dimensionless acceleration $\Gamma$, particles showed either no movement or just spinning on the surface of container. In this experiment four $d=(8.0 \pm 0.2) \mathrm{mm}$ particles were used on center of each wall of the container namely particles 1 and 2 were perpendicular to direction of vibration and particles 3 and 4 were horizontal to direction of vibration as shown in Fig. 1. Particles 1, 2 showed helical movement and the particles 3, 4 showed straight-line movement. Direction of motion of these particles was influenced by the frequency $f$, amplitude $A$, and dimensionless acceleration $\Gamma$.

Three different typed convection patterns were observed at low frequencies $f$ and low dimensionless accelerations $\Gamma$. "Homogeneous convection rolls", "lower-right diagonal convection roll" and "upper-right diagonal convection roll" are shown in Fig. 2.

When system vibrated horizontally with dimensionless acceleration $\Gamma$ less than 1.0 then no movement of particles was observed. The starting critical dimensionless acceleration $\Gamma$ was 1.0 which meant that starting movement of particles depended on provided acceleration $A \omega^{2}$.

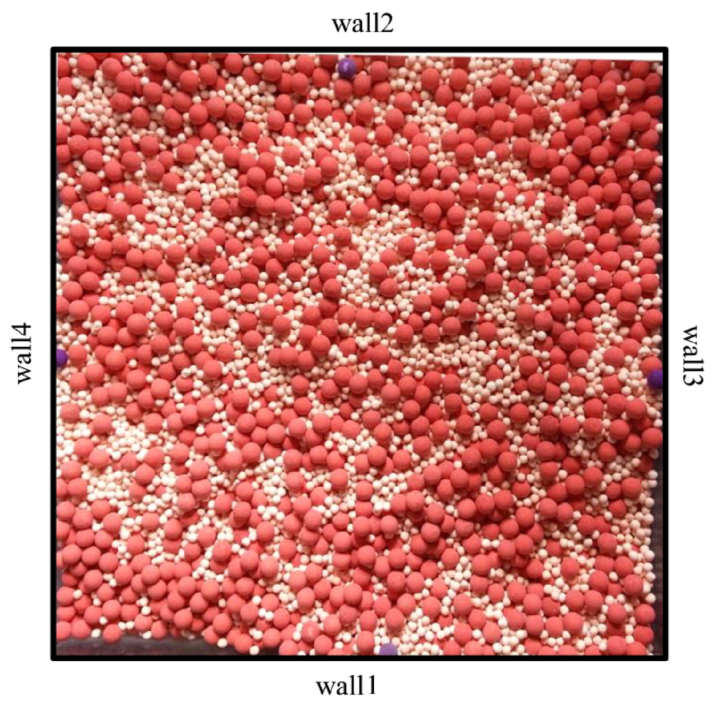

Fig. 1. Surface view of container, indigo colored particles on the middle of walls $1,2,3$ and 4 represent the particles $1,2,3$, and 4 , respectively.

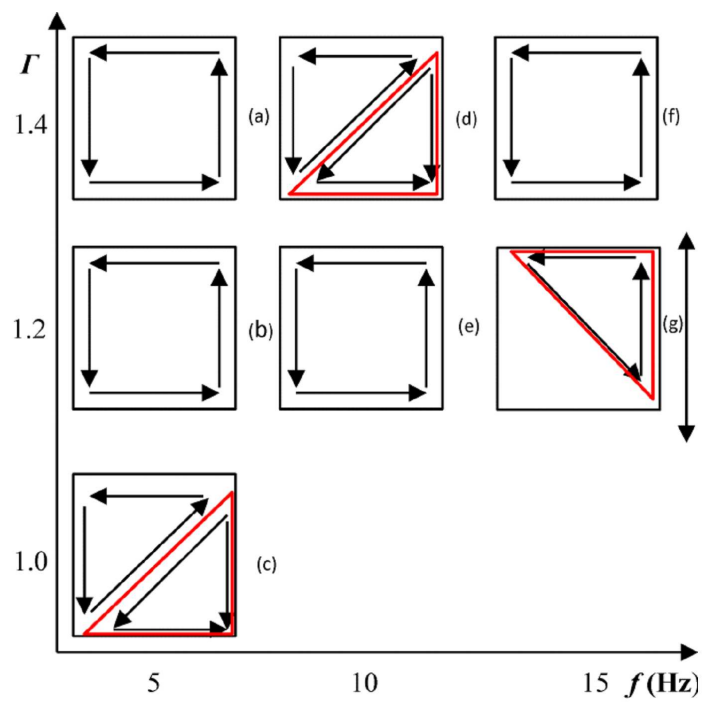

Fig. 2. Surface view of container, (a, b, e, f) represent homogeneous convection rolls, (c, d) show lower right diagonal convection rolls, and (g) represents upper right diagonal convection roll.

It should be equal or greater than gravitational acceleration $g$ :

$$
\begin{aligned}
& \Gamma=a / g=A \omega^{2} / g=1, \\
& a=A \omega^{2}=g .
\end{aligned}
$$

Particles convection was started when the product of amplitude $A$ and frequency $\omega^{2}$ were $A \omega^{2}=g$ and dimensionless acceleration $\Gamma=1.0$.

When $f=5 \mathrm{~Hz}$ and dimensionless acceleration $\Gamma=$ 1.0, then opposite directional helical movement of large particles on walls 1 and 2 was observed, and straight line same directional movement was observed on walls 3 and 4, as a result "lower-right diagonal convection roll" was observed as shown in Figs. 2c and 3c. When dimensionless acceleration $\Gamma$ varied from 1.2 to 1.4 then large 
particles on the walls horizontal to direction of vibrations moved in the opposite direction to each other, therefore, "homogeneous convection roll" was observed as shown in Figs. 2a,b, 3a,b, respectively.

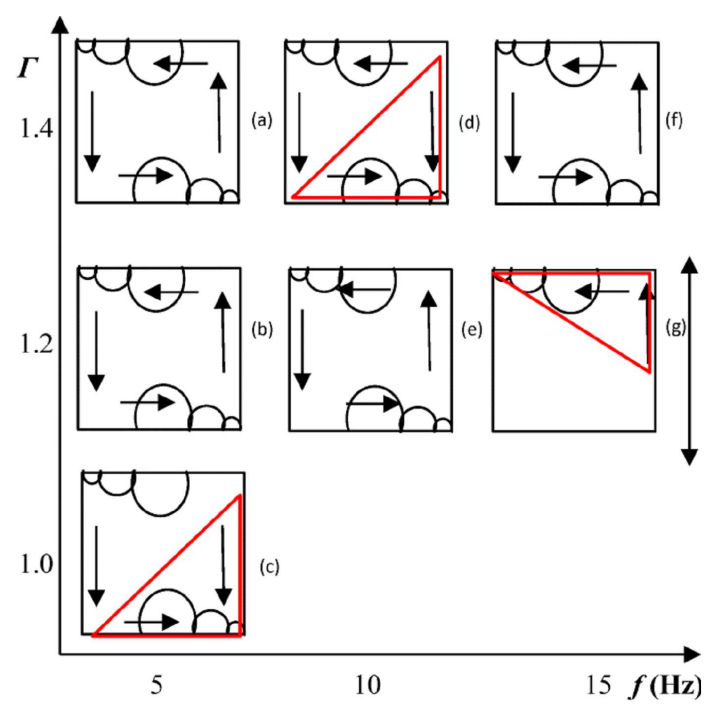

Fig. 3. Movements of individual particles along the walls of container at $5,10,15 \mathrm{~Hz}$ frequency and dimensionless acceleration $\Gamma=1.0,1.2$ and 1.4, arrows represent the direction of motion of particles.

At $10 \mathrm{~Hz}$ frequency no movement of particles was observed before dimensionless acceleration $\Gamma=1.2$. When dimensionless acceleration was $\Gamma=1.2$ then opposite direction helical movement of large particles on wall 1 and 2 were observed and straight line opposite directional movement of large particles on wall 3 and wall 4 were observed. As a result, "homogeneous convection roll" appeared as shown in Figs. 2e and 3e and when dimensionless acceleration $\Gamma=1.4$ then large particles on the walls horizontal to direction of vibrations were moved in the same direction therefore "lower-right diagonal convection roll" was observed as shown in Figs. 2d and 3d.

When system vibrated horizontally with $15 \mathrm{~Hz}$ frequency, then again two types of convection patterns were observed. When dimensionless acceleration $\Gamma$ was 1.2 then movement of large particles was observed only on wall 1 and wall 4 . On wall 1 helical movement of large particles was observed while on wall 4 large particles moved in straight line and toward the wall 1 . Movement was observed only in the half part of container as a result "upper-right diagonal convection roll" observed as shown in Figs. 2g and 3g. When dimensionless acceleration $\Gamma=1.4$ helical movement on the walls 1 and wall 2 and straight line opposite directional movement on the walls 3 and 4 were observed. Therefore, "homogeneous convection roll" observed as shown in Figs. $2 \mathrm{f}$ and $3 \mathrm{f}$.

When the system vibrated, large particles showed helical movement and moved toward the opposite corners of walls $(1,2)$ as shown in Fig. 2, whereas on walls $(3,4)$ large particles moved in a straight line on surface of con- tainer. As the frequency $f$ and dimensionless acceleration $\Gamma$ increased then large particles on walls $(3,4)$ moved in a straight line on the surface whereas on wall $(1,2)$ large particles moved in a helical path (arrows represents the direction of motion of large particles as shown in Figs. 2, 3).

As discussed earlier in Figs. 2 and 3, mostly movement of large particles remained on the walls or corners of container and small particles remained at the center of container. When the system was vibrated, a heap was created in system, position of heap depends on the frequency $f$ and dimensionless acceleration $\Gamma$. When the heap was created at center of container, at that time large particles were moved towards the walls of container and uniformly distributed on the walls of container, therefore, homogeneous convection roll was observed as shown in Fig. 4a,b,e,f. When the heap was shifted towards the wall 1 and wall 3 , at that moment large particles were moved towards wall 2 and wall 3 and a very few particles were moved towards wall 1 and wall 4 , consequently lower right diagonal convection roll was observed as shown in Fig. 4c,d. When the heap was created towards wall 1 and wall 3 , then large particles moved towards wall 2 and wall 3 , at that time upper right diagonal convection roll was observed as shown in Fig. $4 \mathrm{~g}$. When no heap appeared in the container then small and large particles were distributed uniformly in the container. Therefore, no convection rolls were observed as shown in Fig. 4h,i.

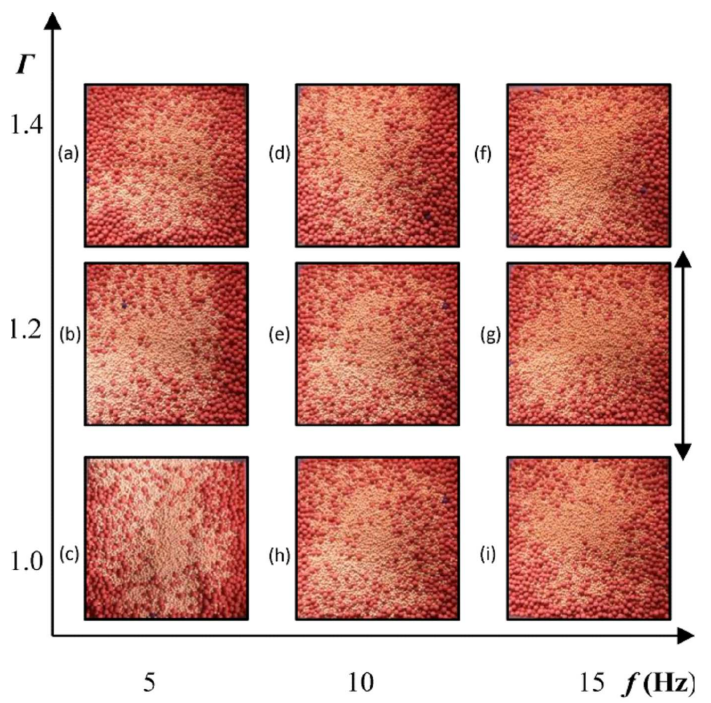

Fig. 4. Surface view of container, final patterns of particles.

\section{Discussion}

When the system was vibrated, large particles along the walls perpendicular to direction of vibration $(1,2)$ moved toward the opposite corners of container as shown in Fig. 3 and path followed by these large particles was helical and this helical behavior is the part of overall convection. When the value of $\Gamma \geq 1$ then at first, particles strike with walls perpendicular to direction of vibration 
$(1,2)$ and after collision of particles with perpendicular walls $(1,2)$ all particles moved toward the center of container and a heap was created as shown in Fig. 5.

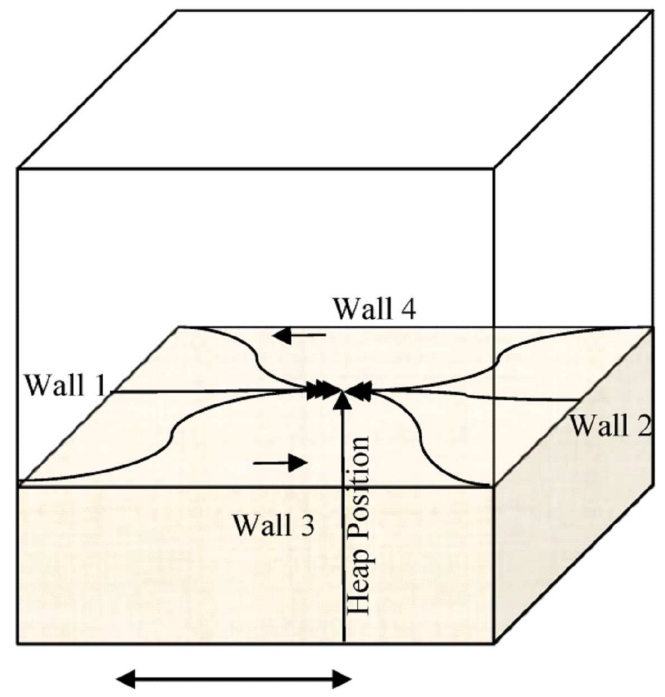

Fig. 5. Particles movements towards the center of container. Vertical arrow represents the heap position; horizontal arrow represents the direction of vibration. Opposite directional movement observed on wall 3 and wall 4.

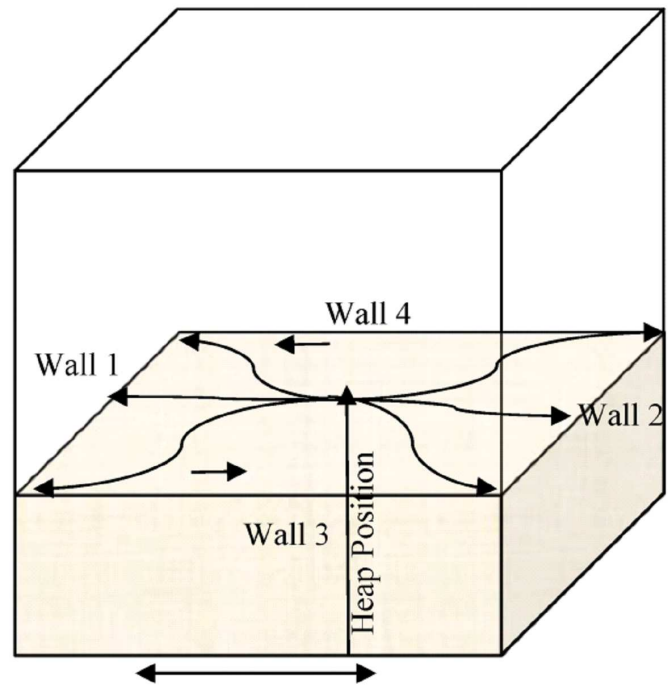

Fig. 6. Particles movement towards the walls of container. Vertical arrow represents heap position; horizontal arrow represents the direction of vibration. Opposite directional movement of particles was observed on wall 3 and wall 4

When the position of heap was at center then large particles moved toward the walls perpendicular to direction of vibration and toward the corners of container. Gaps were observed between walls perpendicular to direction of vibration and particles due to collisions of particles and walls $(1,2)$. Gap spread greater as increase in dimensionless acceleration $\Gamma$, and these gaps filled by the large particle coming from the center of container as shown in Fig. 6.

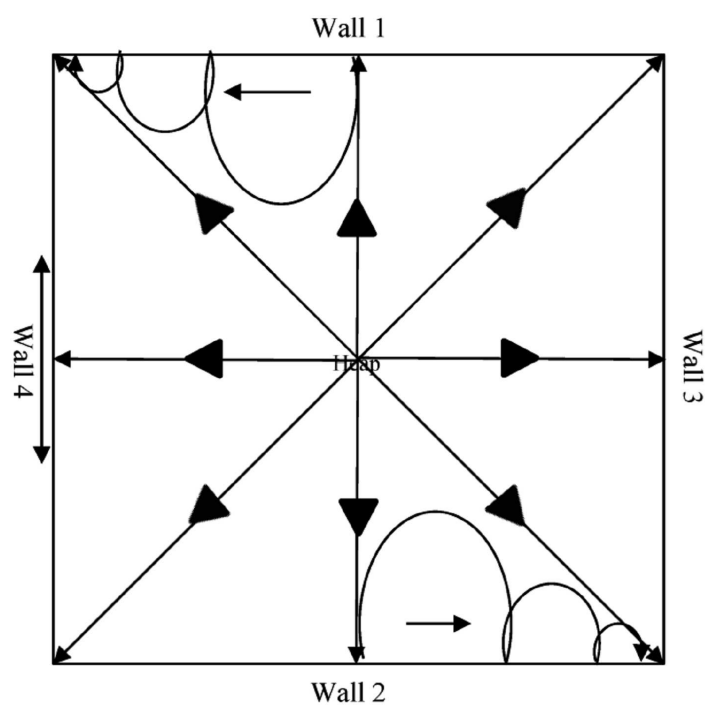

Fig. 7. Central point represents the heap position and arrows from center represent the direction of force applied by large particles moving from heap to perpendicular walls and corners of container.

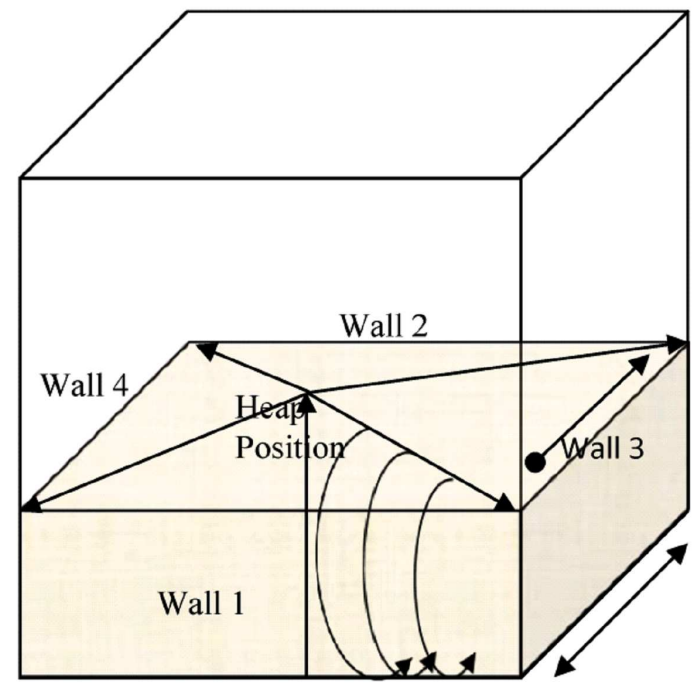

Fig. 8. 3D view of particles movements along the walls 1 and 3. Vertical arrow represents the position of heap. Horizontal arrow represents direction of vibration. Arrows towards the corner of walls represents the direction of force applied by large particles coming from heap. Particles on wall 1 sink downward move straight on the base of container and appear again on the surface. Particles on wall 3 showed straight-line movements.

When large particles fill the gaps between walls perpendicular to direction of vibration $(1,2)$, they move downward and sink. After collision with the base and perpendicular walls, large particles moved straight towards the center of container and appeared again on the surface. Large particles coming from the center of container moved towards walls perpendicular to motion and corners of container exerted forces on the appearing large particles near the walls perpendicular to direction of 
motion. As the result of forces exerted by the large particles coming from the center of container, the appearing large particles change the direction of motion and follow helical path as shown in Fig. 7 and Fig. 8.

On the walls horizontal to the direction of vibration straight line an opposite directional motion was observed. Straight line opposite directional movement of large particles was due to opposite direction helical movement of large particles on walls perpendicular to direction of motion as shown in Fig. 9. When the large particles on walls $(1,2)$ moving toward the corners of container exerted a force on the large particles on walls $(3,4)$, therefore, particles on walls $(3,4)$ moved in opposite direction to each other. Straight-line motion on walls horizontal to direction of motion was due to unavailability of gaps between the walls $(3,4)$ and particles. Therefore, particles did not sink downward as observed on wall $(1,2)$ and no helical movement was observed on wall horizontal to the direction of vibration $(3,4)$.

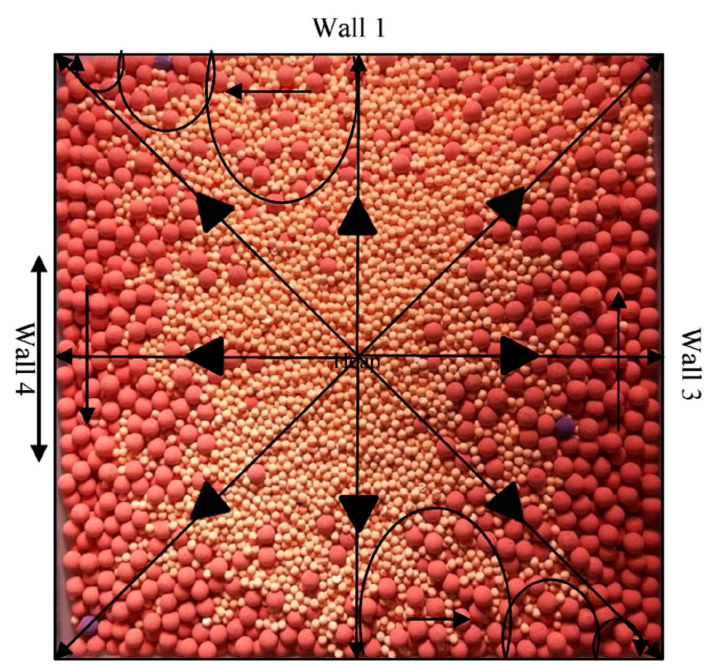

Wall 2

Fig. 9. No gaps present between particle and horizontal wall 3 and wall 4 therefore, particles exert force on each other. Big arrows represent direction of force applied by large particles form wall 1 and wall 2 and small arrows represent the direction of motion of large particles moving from heap.

Three different types of convection rolls were observed "homogeneous convection roll", "lower-right diagonal convection roll" and "upper-right diagonal convection roll". Properties of static heaping and convection depend on $\Gamma[20]$. Large particles showed the same directional movement on the walls horizontally to the direction of motion, then heap was shifted toward wall 2 and 3 at $(f=5 \mathrm{~Hz}$, $\Gamma=1.0)$ and at $(f=10 \mathrm{~Hz}, \Gamma=1.4)$ as shown in Fig. 10. The same directional motion on the walls horizontal to direction of motion was due to the particles moving from heap. Convection depends on frequency and the amplitude of the vibration [22]. Here convection also depends on the frequency and dimensionless acceleration.

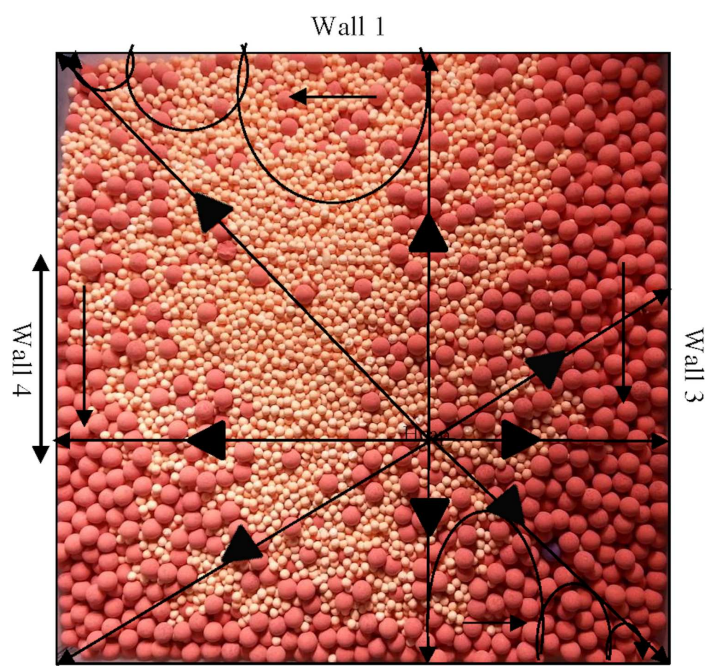

Wall 2

Fig. 10. "lower-right diagonal convection". Heap positon shifted toward the wall 2 and wall 3 . Arrows from heap represent the direction of force applied by large particles moving from heap to perpendicular walls and corners of container.

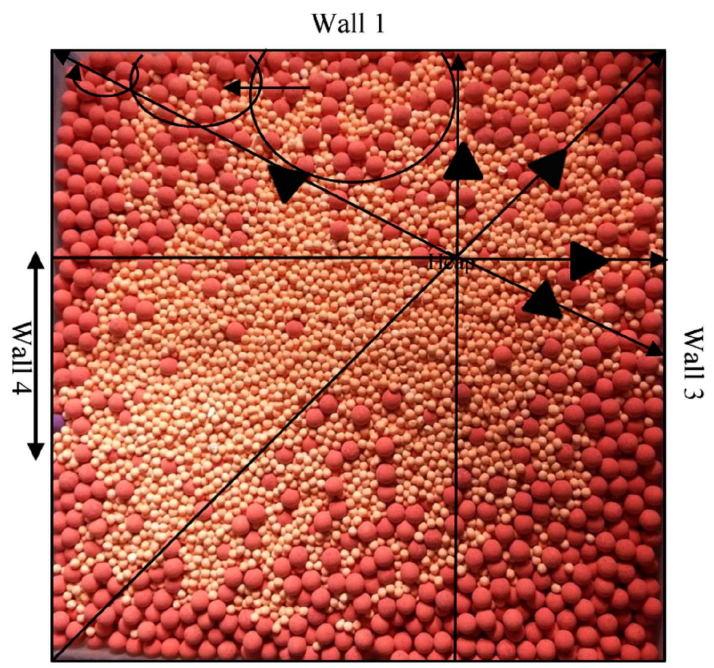

Wall 2

Fig. 11. Surface view of container, "upper right diagonal convection roll" heap shifted towards wall 1 and wall 3. Arrows from heap represents the direction of force applied by large particles moving from heap to perpendicular walls and corners of container.

When $f=15 \mathrm{~Hz}$ and $\Gamma=1.2$, then "upper-right diagonal convection" was observed as shown in Fig. 2 and 3 and it is a result of shifting the heap toward walls 1 and 3 as shown in Fig. 11.

Heap position of particles plays an important role in convection roll mode. As frequency and dimensionless acceleration of particle changes, position of heap also changes as a result convection mode also changes. Table I shows the relation of frequency, dimensionless acceleration, velocity, kinetic energy, heap position, and mode convection. 
TABLE I

Relation between frequency, dimensionless acceleration, convection roll, and heap position.

\begin{tabular}{|c|c|c|c|c|c|}
\hline $\begin{array}{c}\text { Frequency } \\
{[\mathrm{Hz}]}\end{array}$ & $\begin{array}{l}\text { Dimensionless } \\
\text { acceleration } \Gamma\end{array}$ & $\begin{array}{l}\text { Heap } \\
\text { position }\end{array}$ & $\begin{array}{l}\text { Convection } \\
\text { rolls }\end{array}$ & $\begin{array}{c}V=\Gamma / 2 \pi f \\
{[\mathrm{~m} / \mathrm{s}]}\end{array}$ & $\begin{array}{c}E_{k}=m v^{2} / 2 \\
{[\mathrm{~kJ}]}\end{array}$ \\
\hline 5 & 0.8 & & no movement & 9.7 & 95 \\
\hline 5 & 1.0 & toward wall 2,4 & lower right & 7.9 & 63 \\
\hline 5 & 1.2 & centre & homogeneous & 6.4 & 41 \\
\hline 5 & 1.4 & centre & homogeneous & 5.5 & 30 \\
\hline 10 & 0.8 & & no movement & 20 & 386 \\
\hline 10 & 1.0 & & no movement & 16 & 247 \\
\hline 10 & 1.2 & centre & homogeneous & 13 & 170 \\
\hline 10 & 1.4 & toward wall 2,4 & lower right & 11 & 124 \\
\hline 15 & 0.8 & & no movement & 29 & 863 \\
\hline 15 & 1.0 & & no movement & 24 & 555 \\
\hline 15 & 1.2 & toward wall 1,4 & upper right & 20 & 386 \\
\hline
\end{tabular}

Ratios of frequency and dimensionless acceleration are equivalent to velocities of particles given by the system as shown below

$$
\begin{aligned}
& \frac{f}{\Gamma}=\frac{f}{\frac{A \omega^{2}}{g}}, \\
& \frac{f}{\Gamma}=\frac{f g}{V \omega},
\end{aligned}
$$

where $A \omega=V$ (given velocity by the system)

$$
\begin{aligned}
\frac{\omega}{\Gamma} & =\frac{g}{V}, \\
V & =\frac{g \Gamma}{\omega}, \\
V & \propto \frac{\Gamma}{\omega} .
\end{aligned}
$$

Velocity applied to system is directly proportional to $\Gamma / \omega$. Vibrated system applied force on the particles and as force increases velocity of particle increases as shown by equation below

$$
E_{k}=1 / 2 m V^{2},
$$

where $V=\omega / \Gamma$ and $E_{k}=F d$ :

$$
\begin{aligned}
& E_{k}=\frac{1}{2} m(\omega / \Gamma)^{2}, \\
& F={\frac{m^{2}}{\omega}}^{2} \mathrm{~d} \Gamma^{2} .
\end{aligned}
$$

Above equations show that velocity plays an important role on the convection of particles and have direct relationship with the external force applied on the particles. There are certain ranges of velocities, which affect the convection rolls. When $V=\Gamma / \omega=5.5-6.5 \mathrm{~m} / \mathrm{s}$ and $V=\Gamma / \omega=11.5-13 \mathrm{~m} / \mathrm{s}$ heap was created at the center of container then there is most probable chance of homogeneous convection, when $V=\Gamma / \omega=7.5$ $11.5 \mathrm{~m} / \mathrm{s}$ then lower right diagonal convection, and when $V=\Gamma / \omega=11-19.5 \mathrm{~m} / \mathrm{s}$ upper right diagonal convection was observed. Position of heap is function of frequency and dimensionless acceleration and mode of convection is function of heap as shown in table and equations above.

\section{Conclusion}

This research work discusses three types of convection rolls: "homogeneous convection roll", "lower-right diagonal convection roll" and "upper-right diagonal convection roll" on low frequencies and low dimensionless accelerations. These new types of rolls may give new direction to understand the mechanics behind the movement of particles in granular particles systems. Helical movement of large particles was observed along the walls perpendicular to direction of vibration while straight-line movement was observed on walls horizontal to direction of vibration. Direction of large particles along the walls horizontal to direction of vibration influence the convection modes. When $(f=5 \mathrm{~Hz}, \Gamma=1.0$ and $f=10 \mathrm{~Hz}$, $\Gamma=1.4)$ particle on walls horizontal to direction of vibration moved in the same direction, "lower-right diagonal convection roll" appeared. When $(f=15 \mathrm{~Hz}, \Gamma=1.2)$ on one of the wall horizontal to direction of vibration, particles showed no movement while on the other walls which is also horizontal to direction of vibration particle showed straight line movement, as a result "upper-right diagonal convection roll" was observed. These rolls are outcome of heap position. When the heap was at center then "homogeneous convection roll" was observed and when the heap moved from center then other convection rolls were observed, therefore convection rolls depend on the position of heap.

\section{Acknowledgments}

National Natural Science Foundation of China (51476009) sponsored this article.

\section{References}

[1] A.P.J. Breu, H.-M. Ensner, C.A. Kruelle, I. Rehberg, Phys. Rev. Lett. 90, 014302 (2003).

[2] Y. Nahmad-Molinari, G. Canul-Chay, J.C. RuizSuárez, Phys. Rev. E 68, 041301 (2003).

[3] Yan, X. Shi, Q. Hou, M. Lu, K. Chan, C. K. Phys. Rev. Lett. 91, 014302 (2003). 
[4] M.A. Naylor, M.R. Swift, P.J. King, Phys. Rev. E 68, 012301 (2003).

[5] M. Schröter, S. Ulrich, J. Kreft, J.B. Swift, H.L. Swinney, Phys. Rev. E 74, 011307 (2006).

[6] D.C. Hong, P.V. Quinn, S. Luding, Phys. Rev. Lett. 86, 3423 (2001).

[7] J.A. Both, D.C. Hong, Phys. Rev. Lett. 88, 124301 (2002).

[8] J.T. Jenkins, D.K. Yoon, Phys. Rev. Lett. 88, 194301 (2002).

[9] L. Trujillo, M. Alam, H.J. Herrmann, Europhys. Lett. 64, 190 (2003).

[10] M.A. Scherer, V. Buchholtz, T. Pøschel, I. Rehberg, Phys. Rev. E 54, R4560 (1996).

[11] T. Schnautz, R. Brito, C.A. Kruelle, I. Rehberg, Phys. Rev. Lett. 95, 028001 (2005).

[12] S. Tennakoon, L. Kondic, R. Behringer, Europhys. Lett. 45, 470 (1999).

[13] G. Metcalfe, S.G.K. Tennakoon, L. Kondic, D.G. Schaeffer, R.P. Behringer, Phys. Rev. E 65, 031302 (2002).

[14] S. Aumaitre, C.A. Kruelle, I. Rehberg, Phys. Rev. E Stat. Nonlin. Soft Matter Phys. 64, 041305 (2001).

[15] Gallas, Jason AC Herrmann, Hans J Pöschel, Thorsten Sokołowski, Stefan J. Statist. Phys. 82, 443 (1996).

[16] M. Medved, Phys. Rev. E Stat. Nonlin. Soft Matter Phys. 65, 021305 (2002).

[17] S.-S. Hsiau, M.-Y. Ou, C.-H. Tai, Adv. Powder Technol. 13, 167 (2002).
[18] S. Nadler, O. Bonnefoy, J.-M. Chaix, G. Thomas, J.L. Gelet, Eur. Phys. J. E Soft Matter 34, 66 (2011).

[19] K. Liffman, G. Metcalfe, P. Cleary, in: Proc. 3rd Int. Conf. Powders Grains, Eds.: R.P. Behringer, J.T. Jenkins, A.A.Balkema Publishers, Rotterdam 1997, p. 405

[20] S.G. Tennakoon, R. Behringer, Phys. Rev. Lett. 81, 794 (1998).

[21] P. Evesque, Contemp. Phys. 33, 245 (1992).

[22] J. Gallas, H. Herrmann, S. Sokołowski, Phys. Rev. Lett. 69, 1371 (1992).

[23] K. Liffman, G. Metcalfe, P. Cleary, Phys. Rev. Lett. 79, 4574 (1997).

[24] M. Medved, D. Dawson, H.M. Jaeger, S.R. Nagel, Chaos Interdiscipl. J. Nonlin. Sci. 9, 691 (1999).

[25] C. Saluena, T. Pöschel, Europ. Phys. J. E 1, 55 (2000).

[26] I.S. Aranson, L.S. Tsimring, Rev. Mod. Phys. 78, 641 (2006).

[27] F. Rietz, R. Stannarius, Phys. Rev. Lett. 100 , 078002 (2008).

[28] C. Zeilstra, J.G. Collignon, M.A. van der Hoef, N.G. Deen, J.A.M. Kuipers, Powder Technol. 184, 166 (2008).

[29] T. Pöschel, D.E. Rosenkranz, J.A. Gallas, Phys. Rev. E 85, 031307 (2012).

[30] M. Heckel, A. Sack, J.E. Kollmer, T. Pöschel, Phys. Rev. E 91, 062213 (2015).

[31] C. Windows-Yule, New J. Phys. 18, 033005 (2016). 